\title{
Link-based route choice considering risk aversion, disappointment and regret
}

\author{
Achille Fonzone \\ Transport Research Institute, Edinburgh Napier University \\ 10 Colinton Road, Edinburgh, EH10 5DT, UK \\ Phone: +44 (0)131 4552898, Fax: +44 (0)131 4552239 \\ a.fonzone@napier.ac.uk \\ Jan-Dirk Schmöcker \\ Department of Urban Management, Kyoto University \\ C1-2-431, Katsura Nishikyo-ku, Kyoto 615-8540, Japan \\ Phone: +81-75-383-3239, Fax: +81-75-383-3236 \\ schmoecker@trans.kuciv.kyoto-u.ac.jp \\ Jiangshan Ma \\ Department of Civil Engineering, Tokyo Institute of Technology \\ M1-11, 2-12-1, O-okayama, Meguro-ku, Tokyo 152-8552, Japan \\ Phone: +81-3-5734-2577, Fax: +81-3-5734-3578 \\ ma.js@plan.cv.titech.ac.jp \\ Daisuke Fukuda \\ Department of Civil Engineering, Tokyo Institute of Technology \\ M1-11, 2-12-1, O-okayama, Meguro-ku, Tokyo 152-8552, Japan \\ Phone: +81-3-5734-2577, Fax: +81-3-5734-3578 \\ fukuda@plan.cv.titech.ac.jp
}

Words: 6,149

Figures: 6 (1,500 words)

Tables: 1 (250 words)

Total words: 7,899 


\begin{abstract}
In networks with uncertain link travel times route guidance information should consider traveller preferences (1). Risk-averse travellers prefer safer options but might feel unsatisfied if it turns out that their decision has been based on wrong expectations. This paper therefore proposes a new approach to take regret, disappointment and risk aversion into consideration in route navigation. Limited information is assumed: The router knows minimum and maximum travel times of links but not the probability distribution of link travel times. Routes using only potentially optimal links are chosen by weighting the travel times scenarios expected on a given path and taking into account the cost of alternative options. Two approaches are explored, referred to as global and local search. The local search can be implemented as a fast method avoiding path search by guiding travellers link by link. As a drawback local search potentially can lead to cyclic paths. Possibilities to overcome the cyclic path issue and boundary properties of both approaches are discussed. Monte Carlo simulation on a US network suggests that the two approaches can provide good solutions to the problem of route choice under severe lack of information. The local search seems to be particularly promising.
\end{abstract}




\section{INTRODUCTION}

The search for the shortest path (SP) is central to transportation research. SP search becomes non-trivial in networks characterised by uncertainty. Uncertainties in the network conditions might be due to a large number of reasons as extensively discussed in the network reliability literature (see e.g. (2)). It is commonly assumed that the decision maker deals with uncertainty evaluating the likelihood of events as well as their possible duration and impact. Such a complex probabilistic approach requires though extensive information which sometimes is not available. Moreover independence of events is generally assumed which is unrealistic in most cases.

Applications might be divided into descriptive and normative ones. Descriptive ones try to replicate or predict paths taken by individuals. Prescriptive ones are important for route guidance applications and are the focus of this paper. Depending on the application, approaches to SP search often differ. In descriptive applications, such as equilibrium based assignment, one might get away with "rough estimations" and stochastic approaches as the aggregation of a large number of travellers often evens out individual differences in perceptions about the SP. However in prescriptive approaches this is not the case and there is an increasing effort also by navigation device manufacturers to consider individual preferences in detail. Kaparias (3) reports that providing route alternatives is one of the most important criteria for the satisfaction with navigation devices. Furthermore, in contrast to route search for descriptive approaches, route guidance approaches need to give ad hoc advice on what route is best to take given a specific departure time and considering future network conditions. Therefore, route search for route guidance needs to be fast in order to be useful for dynamic applications. These reasons summarise the motivation for this study. The aim is to develop a fast route search algorithm that considers the evaluation of uncertainty by travellers in detail.

The paper is organised as follows: Previous solutions to route guidance and traveller preferences are reviewed in the following section. In contrast to most previous work the approach presented in this paper does not assume that travellers have information on link probability distributions. In line with previous research on risk aversion two approaches are then presented considering travellers' perception of frustration (including disappointment and regret), termed local and global search. Boundary conditions and results for the choice between two links are discussed. In the final part of this paper, for demonstration purposes, an application to the US network is presented before conclusions are drawn.

\section{LITERATURE REVIEW}

In route guidance applications often the computationally efficient $A^{*}$ algorithm is applied, which utilises "node potentials" to reduce the search area (4). These node potentials are set equal to the perceived current estimates of the remaining travel cost when the node is reached. Both "perceived" and "current" though leave open a large area of research.

Firstly, node potentials might not be unique if potential delays vary randomly over time. A significant set of literature has been developed concerning dynamic SPs either as discrete time problem as in Pallotino and Scutellà (5) or continuous time problem as in Ding et al. (6). As arguably the free flow travel time and the potential delay one might encounter are of primary interest, a large number of authors have further looked into the robust SPs problem (7). Significant work has been carried out on finding least expected travel time paths. Hall (8) considers time-invariant stochastic networks and shows that adaptive strategies reduce expected travel time. Miller-Hooks and Mahmassani $(9,10)$ consider the case of stochastic time-dependent networks and develop criteria for a priori SPs as well as algorithms that adapt en-route. The problem quickly becomes too complex to be solved exactly, especially if multiple criteria are used to determine SPs as in Nielsen et al. (11).

Secondly, different "perceived node potentials" can lead to different optimal paths. Golledge and Gärling (12) comprehensively summarise previous research and discuss human factors that influence travel decisions in general and more particularly routing decisions. Besides shortest travel time and distance Golledge and Stimson (13) find criteria such as minimizing turns, driving the longest part of the journey first, defining travel corridors, heading always in the direction of the destination or route aesthetics to be important. Some of these preferences might be reflected in a SP algorithm by adding 
penalties to turning links or links that do not point in the direction of the destination, but other preferences are more difficult to be included.

To give good advice to travellers under limited information, the decision device needs to understand user's decision principles. Three decision criteria are mainly discussed in the normative literature on decision making: optimism, pessimism and regret (14). Regret is experienced when realizing that a better outcome could have been achieved if a different decision had been taken. The regret perception in choice scenarios is associated with the work of Savage (15). Research has proven that disappointment plays an important role in decision making as well $(16,17)$. Similarly to regret, disappointment derives from "counterfactual thinking" (18). It arises when the actual realization of an event generates an outcome worse than the decision maker's prior anticipation. Despite being long-known in the psychological field, disappointment is usually neglected in decision making models. In most cases none of the previous criteria will be applied by travellers in their pure form but several principles will play a role in mode or route choice decisions. Especially introducing regret has been of recent interest for transport researchers. Chorus et al. (19) describe a random regret minimization approach within a discrete choice framework. The decision maker considers the potential regret of the current choice with respect to the best available option for each scenario and applies this to mode choice analysis with risky travel times and costs. Chorus (20) extends this work by introducing a regret function with respect to all forgone options and a smooth likelihood function for econometric applications. He finds that in general the random regret minimization approach outperforms the random utility approach without consideration of regret. Chorus (21) studies traffic equilibria considering regret and risk-aversion through numerical examples. The author concludes that ignoring regret aversion may lead to overestimation of flows on risky paths.

To consider user preferences and decision criteria within route navigation Park et al. (1) develop a module that learns preferences whenever the user is taking a route different from the recommended one. Attributes of the alternative route are stored and given higher priority next time a route is chosen. Partly avoiding these issues of preferences and decision criteria Kaparias et al. (22) develop a navigation approach suggesting a driver multiple disjoint paths. Suggesting multiple paths leaves the drivers with the problem of selecting a single path, before, or at latest when approaching, decision points where the paths split. In line with Kaparias et al. (22) the Hyperstar approach of Bell (23) suggests a set of routes which include in particular safe options when travellers are afraid that some links might be delayed. To decide the actual path Bell suggests taking the link at each junction with the highest probability of being the shortest.

In the following an approach is developed incorporating regret and disappointment. Similarly to Bell (23) only minimal information is assumed available on the state of the network.

\section{DECISION MAKING APPROACH}

A framework is proposed and tested for route choice in transport networks in which the travel time of each link can take only two values, corresponding to undelayed and delayed traffic conditions. No information is considered available on the probability of each state. The choice is based on risk-aversion and consideration of disappointment and regret, collectively referred to as frustration.

A two-step decision making process is adopted. Firstly a set of the links which are optimal when going from the origin to the destination in at least one combination of link states is identified. Such a subnetwork and all its elements are defined "potentially optimal" (PO). Subsequently a path is selected that is made up of PO links only. This first stage has been presented in (24) whose main results are reported below.

Two link-based approaches to path selection are brought forward. The first interprets expected disappointment and regret as link cost penalties and applies a common SP algorithm using the set of modified costs including disappointment and regret. Since (possibly) all the PO links are considered for the decision, the approach is called "global search". The second approach incorporates expected frustration as a criterion to select iteratively one PO link downstream of the last node included in the path 
identified by the search algorithm until the destination is reached. The method is called "local search" because the choice considers only the links at the node under consideration.

\section{NOTATION AND DEFINITIONS}

Throughout the paper the following notation is used.

$$
\begin{aligned}
& I \quad \text { Set of nodes } \\
& \text { A Set of links } \\
& A_{i}^{+} \quad \text { Forward star of node } i \\
& P_{i}(a) \quad \text { Set of paths from node } i \text { to the destination using } a \in A_{i}^{+} \text {( } a \text { is omitted to } \\
& \text { indicate the set of all the paths from } i \text { to the destination) } \\
& a^{+}, a^{-} \quad \text { Head node, tail node of link } a \\
& r, s \quad \text { Origin, destination } \\
& c_{a}, d_{a} \quad \text { Undelayed cost, potential delay on link } a \\
& \omega \in \Omega \quad \text { Particular realization of link states (undelayed/delayed) within the set } \Omega \text { of } \\
& \phi, \delta \quad \text { Scenarios within set } \Omega \text { in which all links are undelayed, delayed } \\
& p^{*}(\omega) \in P_{i}(a) \quad \text { Optimal path from } i \text { to } s \text { using link } a \in A_{i}^{+} \text {leaving } i \text { under delay scenario } \omega \\
& k_{a}(\omega) \quad \text { Cost of link } a \text { under } \omega: k_{a}(\omega)= \begin{cases}c_{a} & \text { if } \omega=\phi \\
c_{a}+d_{a} \text { if } \omega=\delta\end{cases} \\
& g_{p}(\omega), g_{p}(\bar{\Omega}) \quad \text { Cost of path } p \text { under } \omega, \forall \omega \in \bar{\Omega} \subseteq \Omega \\
& h_{i}(\omega, a) \quad \text { Cost of the optimal path from } i \text { to the destination (node potential of } i \text { ) under } \\
& \omega \text { conditional on using link } a \text { ( } a \text { is omitted to indicate the minimum cost } \\
& \text { under } \omega \text { ) } \\
& \Omega_{i}^{\phi}, \Omega_{i}^{\delta} \quad\left\{\omega \mid h_{i}(\omega)=h_{i}(\phi)\right\},\left\{\omega \mid h_{i}(\omega)=h_{i}(\delta)\right\} \\
& \Omega_{a}^{\phi}, \Omega_{a}^{\delta} \quad\left\{\omega \mid k_{a}(\omega)=k_{a}(\phi)\right\},\left\{\omega \mid k_{a}(\omega)=k_{a}(\delta)\right\}
\end{aligned}
$$

"Optimistic" ("pessimistic") designates the route choice in which for each variable the value it assumes in $\phi(\delta)$ is anticipated. Note that the value assumed by a variable in $\phi$ (or in $\delta$ ) can occur under different $\omega$, e.g. the node potential of node $i$ is equal to $h_{i}(\phi)$ in all the scenarios in which the links of $p_{i}(\phi)$ are undelayed.

Potential optimality is formally defined as follows

Definition 1 (PO path): $p \in P_{i}$ is potentially optimal $\Leftrightarrow \exists \omega \in \Omega \mid h_{i}(\omega)=g_{p}(\omega)$, i.e. a congestion scenario $\omega$ exists such that $p$ is the shortest path from $i$ to $s$.

Definition 2 (PO link): $a$ is potentially optimal $\Leftrightarrow \exists \omega \in \Omega, p \in P_{i}(a) \mid h_{a^{-}}(\omega)=g_{p}(\omega)$, i.e. there is at least one potentially optimal path from the tail of node $a$ to $s$ including $a$.

Definition 3 (PO node): $i$ is potentially optimal $\Leftrightarrow \exists$ potentially optimal $a \in A$ such that $i=a^{+}$ or $i=r$, i.e. a node $i$ is potentially optimal if it is the head of a potentially optimal link or is $r$.

\section{POTENTIALLY OPTIMAL NETWORKS}

A network with $|A|$ links, each of which can be either undelayed or delayed, can assume $2^{|A|}$ different states. Potentially each network state has a different SP connecting the same OD pair. Although the 
number of the possible SPs can be huge, some links might never be included in a SP which means they are non-PO. It is assumed that

Assumption 1: Non-PO links are not considered in route choice and so path search is confined to the PO network.

It can be proven that

Proposition 1 (Necessary condition for $a$ to be PO): $a$ is $\mathrm{PO} \Rightarrow h_{a^{-}}(\phi, a)<h_{a^{-}}(\delta, b) \quad \forall b \neq a \in A_{a^{-}}^{+}$

that is, if a link $b$ exists leaving from the same tail node as $a$ such that $b$ is more convenient than $a$ to reach the destination even when the network condition is most favourable to $a$ and most adverse to $b$, then $a$ is never optimal.

The condition in Proposition 1 is not sufficient for $a$ to be PO because of the possibility of overlaps between the optimistic SP (OptSP) from $a^{+}$and the pessimistic one (PesSP) from $b^{+}$. Therefore using this condition only allows identifying a set of links which might be PO (and its complement, a set of links which are non-PO). Other conditions are required to decide on the actual potential optimality of such links. The following can be derived

$$
\begin{aligned}
& \text { Proposition } 2 \text { (Sufficient condition for a to be PO): } \\
& k_{a}(\phi)<\max \left\{k_{b}(\delta)+h_{b^{+}}(\phi)-h_{a^{+}}(\phi), k_{b}(\delta)+h_{b^{+}}(\delta)-h_{a^{+}}(\delta)\right\} \forall b \neq a \in A_{a^{-}}^{+} \Rightarrow a \text { is potentially optimal }
\end{aligned}
$$

Note that in contrast to literature on robust SP, Proposition 2 identifies a set of PO links and not a single path. Proposition 2 allows identifying only a subset of all PO links of a network ("PO network" hereafter). The fraction of the set of PO links provided by Proposition 2 cannot be evaluated exactly, since the problem of finding all PO links with an exhaustive search becomes rapidly intractable as $|A|$ increases. Monte Carlo simulation can be used as an alternative method to identify PO links. Comparison between Proposition 2 and Monte Carlo simulation shows that the two approximate approaches provide comparable information, but the implementation of Proposition 2 is much faster and tends to recognize more PO links when running time constraints are imposed (24). Remarkable is also that the set of PO links identified by Proposition 2 includes OptSP and PesSP.

\section{ROUTE CHOICE CONSIDERING RISK-AVERSION AND FRUSTRATION}

In the context of navigation assistance a good method to select a path from the PO network ought to comply with the following requirements: It should

- utilize all the knowledge already developed in defining the PO network to ensure fast calculation and to avoid wasting information.

- consider that a non-optimal choice can be easily made with so scarce information, therefore the traveller can experience frustration.

- account for different levels of optimism/pessimism.

To achieve these requirements in this paper two methods are defined and evaluated based on two preference assumptions:

Assumption 2 (risk-aversion): When evaluating the cost of $x \in\{I, A\}$, the occurrence of the pessimistic condition is anticipated with probability $\mathrm{f}_{\text {pes }}(\boldsymbol{\alpha} ; \chi)$ and that of the optimistic condition with probability $1-\mathrm{f}_{\text {pes }}(\boldsymbol{\alpha} ; \chi)$. 
Where $\boldsymbol{\alpha}$ is a set of parameters representing the level of risk-aversion and $\mathrm{f}_{\mathrm{pes}}(\boldsymbol{\alpha} ; \chi)$ is defined so that $\mathrm{f}_{\text {pes }}(\mathbf{1} ; \chi)=1$. To ensure the speed of the solution, the following simplifications are applied:

- The state of link $a$ and that of its downstream links are independent.

- Possible overlaps of the optimistic and pessimistic paths are not considered.

Assumption 3 (frustration): Route choice is made on the basis of modified link costs including the frustration arising for the chosen link $a$ when the anticipated $\omega$ is such that

- A cost is incurred on $a$ itself greater than that considered in choosing $a$ (disappointment)

- With respect to a concurrent link $b \in A_{a^{-}}^{+}$the optimal path using $b$ is shorter than that using $a$, i.e. when $g_{p^{*}}(\omega)>g_{q^{*}}(\omega), p^{*} \in P_{a^{-}}(a), q^{*} \in P_{a^{-}}(b)$ (regret).

In general consideration of counterfactual emotions can be described in terms of two features:

- Horizontal perspective: "isolated" choices consider exclusively what happens on the examined option, "comparative" ones look also at concurrent options. In "isolated" decision making frustration coincides with disappointment, in the "comparative" one it includes also regret.

- Longitudinal perspective: "short-sighted" choices do not consider possible frustration downstream from the end of the next link, "far-sighted" will.

The presented framework can deal with any combination of horizontal and longitudinal perspectives. Consistent with the objective of exploiting all the available information, in the following comparative far-sighted preferences are analysed.

\section{Definition of expected frustration}

To implement Assumption 3 the choice of $p \in P_{a^{-}}(a)$ is associated with an expected frustration $E\left(f_{a}\right)$ calculated with equations [1]-[4]. The anticipated excess travel time $e_{a x}\left(\Omega_{a}, \Omega_{x}\right), x \in \hat{A}_{a^{-}}^{+}$when link $a$ is chosen is

$$
e_{a x}\left(\Omega_{a}, \Omega_{x}\right)= \begin{cases}0 & \text { if } \hat{A}_{a^{-}}^{+}=\{\} \\ g_{p^{*}}\left(\Omega_{a}\right)-g_{q^{*}}\left(\Omega_{x}\right), p^{*} \in P_{a^{-}}(a), p^{*} \in P_{a^{-}}(x), x \in \hat{A}_{a^{-}}^{+} & \text {otherwise }\end{cases}
$$

Where $\hat{A}_{a^{-}}^{+} \subseteq A_{a^{-}}^{+}$is the set of links to which $a$ is compared. It depends on the assumed decision making process, as explained below in TABLE 1 . The set $\Omega_{y}$ (where $y$ denotes link $a$ or $x$ ) stands for the set of four link cost scenarios $\left\{\left(\Omega_{y}^{\phi} \cap \Omega_{y^{+}}^{\phi}\right),\left(\Omega_{y}^{\phi} \cap \Omega_{y^{+}}^{\delta}\right),\left(\Omega_{y}^{\delta} \cap \Omega_{y^{+}}^{\phi}\right),\left(\Omega_{y}^{\delta} \cap \Omega_{y^{+}}^{\delta}\right)\right\}$. In other words the optimal $p^{*}$ and $q^{*}$ and their cost are found for four cases: 1) $a$ not congested, links downstream of $a^{+}$(the head node of a) not congested; 2) $a$ not congested, links downstream of $a^{+}$congested; 3) $a$ congested, links downstream of $a^{+}$not congested; 4) $a$ congested, links downstream of $a^{+}$congested.

Combining the four $\Omega_{y}$ of each of the two paths the router evaluates sixteen values of $e_{a x}\left(\Omega_{a}, \Omega_{x}\right)$ for each $(a, x)$, assuming that $\Omega_{a}$ and $\Omega_{x}$ can simultaneously exist on the network although this might not be the case because of overlaps. The cost of a $p \in P_{y^{-}}(y)$ can be obtained with 


$$
\begin{aligned}
g_{p}\left(\Omega_{y}\right) & =k_{y}\left(\Omega_{y}\right)+\lambda \cdot h_{y^{+}}\left(\Omega_{y}\right) \\
& =\left(\left(1-\xi_{y}\right) \cdot k_{y}(\phi)+\xi_{y} \cdot k_{y}(\delta)\right)+\lambda \cdot\left(\left(1-\xi_{y^{+}}\right) \cdot h_{y^{+}}(\phi)+\xi_{y^{+}} \cdot h_{y^{+}}(\delta)\right)
\end{aligned}
$$

Where $\xi_{y}$ is 0 if $y$ is not delayed in $\Omega_{y}$ and 1 if it is. Similarly $\xi_{y^{+}}$is 0 if the node potential of $y^{+}$is the optimistic one and 1 if it is the pessimistic one. $\lambda$ is 1 for far-sighted choices and 0 for shortsighted.

A frustration cost can be associated to $e_{a x}\left(\Omega_{a}, \Omega_{x}\right)$

$$
f_{a x}\left(\Omega_{a}, \Omega_{x}\right)=\mathrm{f}_{\text {fru }}\left(\beta ; e_{a x}\left(\Omega_{a}, \Omega_{x}\right)\right)
$$

The frustration of $a$ w.r.t. $x$ over all the sixteen delay scenarios is the average of all $f_{a x}\left(\Omega_{a}, \Omega_{x}\right)$ weighted by the probabilities of $\left(\Omega_{a}, \Omega_{x}\right)$

$$
E\left(f_{a x}\right)=\sum_{\Omega_{a}, \Omega_{x}} f_{a x}\left(\Omega_{a}, \Omega_{x}\right) \cdot\left(\pi\left(\Omega_{a}\right) \cdot \pi\left(\Omega_{x}\right)\right)
$$

The probability $\pi\left(\Omega_{y}\right)$ is not objective but subjective and depends exclusively on the attitude of the decision maker towards risk and frustration. Given Assumption 1 the following holds

$$
\left.\pi\left(\Omega_{y}\right)=\left(\left(1-\xi_{y}\right) \cdot\left(1-\mathrm{f}_{\text {pes }}(\boldsymbol{\alpha} ; y)\right)+\xi_{y} \cdot \mathrm{f}_{\text {pes }}(\boldsymbol{\alpha} ; y)\right) \cdot\left(1-\xi_{y^{+}}\right) \cdot\left(1-\mathrm{f}_{\text {pes }}\left(\boldsymbol{\alpha} ; y^{+}\right)\right)+\xi_{y^{+}} \cdot \mathrm{f}_{\text {pes }}\left(\boldsymbol{\alpha} ; y^{+}\right)\right)
$$

Expected frustration is the mean value of $E\left(f_{a x}\right)$

$$
E\left(f_{a}\right)=\sum_{x \in \hat{A}_{a^{+}}^{+}} \frac{E\left(f_{a x}\right)}{\left|\hat{A}_{a^{-}}^{+}\right|}
$$

Different kinds of route choice can be described using this framework, as shown in TABLE 1. Note that when frustration is considered $a$ is always included in $\hat{A}_{a^{-}}^{+}$. the following

Route choice depends on the specification of $\mathrm{f}_{\text {pes }}$ and $\mathrm{f}_{\text {fru }}$. For $\mathrm{f}_{\text {pes }}$ a constant function is analysed in

$$
\mathrm{f}_{p e s}\left(\alpha_{1}, \alpha_{2} ; y\right)=\left\{\begin{array}{l}
\alpha_{1} \text { if } y \in A \\
\alpha_{2} \text { if } y \in I
\end{array}\right.
$$

Following (25) it is considered

$$
\mathrm{f}_{\text {fru }}\left(\beta ; e_{a x}\left(\Omega_{a}, \Omega_{x}\right)\right)= \begin{cases}0 & \text { if } e_{a x}\left(\Omega_{a}, \Omega_{x}\right) \leq 0 \\ {\left[e_{a x}\left(\Omega_{a}, \Omega_{x}\right)\right]^{\beta}} & \text { otherwise }\end{cases}
$$

This implies that elation is not taken into account (i.e. no positive utility comes from $\left.e_{a x}\left(\Omega_{a}, \Omega_{\chi}\right)>0\right)$.

\section{Using expected frustration in route choice}


Two methods for route choice are proposed based on two different interpretations of the expected frustration $E\left(f_{a}\right)$.

\section{Global search considering frustration penalties}

$E\left(f_{a}\right)$ can be interpreted as a penalty and is added to the anticipated link cost to obtain a modified link cost $k_{a}^{\prime}$. In formulas

$$
k_{a}^{\prime}=E\left(k_{a}\right)+E\left(f_{a}\right)
$$

where

$$
E\left(k_{a}\right)=\left(1-\alpha_{1}\right) \cdot k_{a}(\phi)+\alpha_{1} \cdot k_{a}(\delta)
$$

The minimum cost path can then be identified applying any SP algorithm to the set of the modified costs.

\section{Local search using modified path disutilities}

Following (26), $E\left(f_{a}\right)$ can be considered as a component of the modified disutility of $p^{*} \in P_{a}(\omega)$ :

$$
g_{p^{*}}^{\prime}=E\left(g_{p^{*}}\right)+E\left(f_{a}\right)
$$

where

$$
E\left(g_{p^{*}}\right)=E\left(k_{a}\right)+\left(1-\alpha_{2}\right) \cdot h_{a^{+}}(\phi)+\alpha_{2} \cdot h_{a^{+}}(\delta)
$$

When a node is reached in the path search the following link is selected comparing the disutilities of the available alternative paths. The link included in the path with the smallest disutility is added to the suggested path. Then the head node of the newly added link is examined until the destination is reached. This means, in contrast to global search, no SP search needs to be employed but the SP is "step-wise" discovered by determining always the next link when reaching the next node until the destination is reached.

\section{Discussion of the two methods}

Local search is attractive for navigation assistance applications because of its speed. However it can get stuck in cyclic paths. To deal with this problem an approach is proposed based on the consideration that for a loop to appear links have to be involved backward oriented w.r.t. the direction from the origin to the destination. Backward links can be easily detected in transport networks by looking at the geographical coordinates of nodes. Whenever loops arise backward links are penalized by incrementing their cost by a fixed percentage until a path can be identified from the origin to the destination. The algorithms for global and local searches are summarized below ( $A^{P O}$ indicates the PO links).

\begin{tabular}{|l|l|}
\hline \multicolumn{1}{|c|}{ Global search } & \multicolumn{1}{c|}{ Local search } \\
$\begin{array}{l}\text { 1. Calculate } k_{a}^{\prime} \quad \forall a \in A^{P O} \\
\text { 2. Determine the suggested path by applying any } \\
\text { SP algorithm to } A^{P O} \text { with costs } k_{a}^{\prime}\end{array}$ & $\begin{array}{l}\text { 1. Set } r \text { as the current suggested node } i \\
\text { 2. Calculate } g_{p^{*}}^{\prime}, p^{*} \in P_{x^{+}}(x) \forall x \in A_{i}^{+} \cap A^{P O} \\
\text { 3. Add } \bar{x}=\arg \min _{x \in A_{i}^{+}} g_{p^{*}}, p^{*} \in P_{x^{+}}(x) \text { to the } \\
\text { suggested path }\end{array}$ \\
\hline
\end{tabular}


4. If the addition of $\bar{x}$ generates a loop, increase $k_{x}(\phi)$ and $k_{x}(\delta)$ of every backward link $x$ by $\mathrm{p} \%$ and restart

5. If $\bar{x}^{+}=s$ the search is finished, otherwise set $i=\bar{x}^{+}$and iterate from 2

Some properties of the two methods are illustrated in FIGURE 1 for the case of a node with two departing links. The anticipated link cost is not influenced by anticipations of what happens downstream of the head node, so it does not depend on $\alpha_{2}$. FIGURE 1(a) shows that for $\alpha_{1}=0$ it is equal to the optimistic travel time, for $\alpha_{1}=1$ to the pessimistic one. FIGURE 1(b) illustrates that disappointment, $\mathrm{f}_{\text {fru }}\left(e_{a a}\right)$, is symmetrical with respect to both $\alpha_{1}$ and $\alpha_{2} \cdot \alpha_{1}=\alpha_{2} \in\{0,1\}$ characterizes "confident" decision making in the sense that only one of the sixteen cost scenarios considered in the calculations of $E\left(f_{a b}\right)$ is deemed possible. Hence in these cases disappointment is equal to 0 . The ratio $\frac{\left|\alpha_{i}-0.5\right|}{0.5}, i=1,2$ can be interpreted as "level of certainty" such that higher certainty corresponds to lower feared disappointment.

Regret, $\mathrm{f}_{\text {fru }}\left(e_{a b}\right)=0$ for $b \neq a$, arises when the path using $a$ turns out to be more expensive than that using $b$. When the router is completely optimistic $\left(\alpha_{1}=\alpha_{2}=0\right)$ the router is certain that all the links are undelayed. In such condition OptSP is the best option hence no regret is expected from using $a$. Analogously, when a completely pessimistic view is taken regarding both the next link and what happens downstream after the next link ( $\alpha_{1}=\alpha_{2}=1$ ), the expected frustration is 0 for $b$ (FIGURE 1(c)).

The expected frustration is continuous, non-monotonic w.r.t. $\alpha_{1}$ and $\alpha_{2}$, and monotonically increasing with $\beta$ (FIGURE 1(d, e)). FIGURE 1(e) illustrates the complex interaction between the $\alpha$ and $\beta$ parameters. The sensitivity of expected frustration to pessimism increases with the value attached to frustration, i.e. the higher $\beta$ the higher the variation of expected frustration with $\alpha$.

OptSP and PesSP can be obtained as particular cases of the global search. In fact for $\alpha_{1}=\alpha_{2}=0$ there is no disappointment or regret, so no frustration is associated to the links of OptSP and hence the modified link cost is equal to the anticipated link cost. In contrast for other scenarios regret and frustration are in general different from 0 so the modified cost is higher than the anticipated one. When $\alpha_{1}=0$ the anticipated link costs are equal to the undelayed ones, so the cost of OptSP is the optimistic one whereas other paths are at least as expensive as in the optimistic network status. Since the latter paths are already more expensive than OptSP when their cost is the optimistic one, a fortiori OptSP is the most convenient path. Similarly it can be proven that PesSP is suggested by the global search with $\alpha_{1}=\alpha_{2}=1$.

Also, given the definition of modified path disutility and the observations on expected frustration, the local search chooses the links of OptSP for $\alpha_{1}=\alpha_{2}=0$ and those of PesSP for $\alpha_{1}=\alpha_{2}=1$. FIGURE 1(f) illustrates that the more pessimistic views are taken and the higher the importance given to frustration, the more PesSP tends to be chosen.

A peculiarity of the proposed route choice models is shown in FIGURE 2 where $\alpha_{1}=0.8, \alpha_{2}=0.3, \beta=0.65$ have been assumed (this value of $\beta$ is found in discrete choice experiments considering expression of frustration similar to that proposed in (25)). In terms of input data $\left(k_{a}, h_{i}\right)$ the two networks are equivalent in the sense that for both $h_{i}(\phi)=55, h_{i}(\delta)=100$, OptSP from $i$ is that using link $a$ and PesSP that using link $b$. Therefore network 2 could be obtained by network 1 by adding $j$ as a fictitious node. The anticipated link costs on $b$ and $b^{\prime}+b^{\prime \prime}$ are the same but the frustration penalties and hence the modified costs are different. As a consequence the local search chooses Link $a$ in network 1 and $b$ ' in 2. The global search is affected as well. This means that route choice is influenced by the network description. In terms of the classical utility theory this is a drawback. However the fact that $k_{b}^{\prime}<k_{b^{\prime}}^{\prime}+k_{b^{\prime \prime}}^{\prime}$ 
resembles the phenomenon of lower subadditivity of weighting function, which is well-known in the field of decision under uncertainty for events with low objective probability (27).

\section{MONTE CARLO EVALUATION}

SP found by global and local search can be considered as possible heuristic solutions to a "problem with unknown structure" (28). They provide a priori SPs (respectively GloSP and LocSP) in the sense that they are calculated before knowing the link status. OptSP and PesSP also can be considered $a$ priori heuristic solutions. The quality of the heuristic methods can be evaluated comparing their solutions to the actual SP in the simulation (SimSP) which can be calculated after the scenario of link delays has been set (this scenario is assumed unknown to the decision maker at the point of decision making). The Monte Carlo simulation approach described in FIGURE 3 is used for this purpose. Each experiment in a Monte Carlo trial is characterized by a specific realization of link states. The state of a link is randomly defined by extracting a number from a uniform distribution with support $[0,1]$ and considering the link undelayed if the number is smaller than 0.5 , delayed otherwise. The topological similarity of two paths is measured by a similarity index

$$
S I=\frac{\sum_{a \in A} x_{a} \cdot y_{a}}{\sqrt{\sum_{a \in A} x_{a}^{2}} \cdot \sqrt{\sum_{a \in A} y_{a}^{2}}}
$$

where $x_{a}\left(y_{a}\right)$ is 1 if Link $a$ is part of the first (second) path, 0 otherwise. $S I=1$ for identical paths, $S I=0$ for disjoint ones.

For illustration purpose the network of U.S. highways is used (3796 bidirectional links, 2922 nodes, FIGURE 4(b)). The undelayed travel times $c_{a}^{U S}$ are supposed proportional to the link length (a speed of $60 \mathrm{~km} / \mathrm{h}$ is assumed), the "base" delays are determined by the formula

$$
d_{a}^{\text {USbase }}(\delta)=\rho \cdot c_{a}^{\text {US }}
$$

where $\rho$ is extracted from a continuous uniform distribution with support $(0,1]$.

1,000 link state draws have been carried out for each of nine trials. In trial 1-5 the origin is fixed at New York and five destinations are considered at increasing Euclidean distance: Detroit (568 km), Chicago (954 km), Kansas City (1,603 km), Salt Lake City (2,958 km), Los Angeles (3,745 km) (FIGURE 4(a)). Base delayed travel times are used. In trials 6-9 the SPs are calculated for the same OD pair (New York - Los Angeles) considering increasing levels of delays. In trial 7 base delays $d_{a}^{\text {USbase }}$ are used, whereas in 6,8 and 9 delays are considered equal to $0.5 \cdot d_{a}^{\text {USbase }}, 5 \cdot d_{a}^{\text {USbase }}$ and $10 \cdot d_{a}^{\text {USbase }} \forall a$. In the frustration-based searches $\alpha_{1}=\alpha_{2}=0.5, \beta=0.65$ is assumed.

FIGURE 4 shows examples of the results of the Monte Carlo simulation. FIGURE 4(b) shows that when the level of the delay which can affect the network increases, the PO network spreads. The SI of LocSP and GloSP in (c) is 0.88 .

FIGURE 5 and FIGURE 6 provide an overview of the performance of the four heuristics for increasing OD distance (trials 1-5) and increasing delay levels (trials 6-9). In (a) the absolute performance of each of the four a priori SPs is reported, in terms of average SI with SimSP. Generally longer distances between origin and destination and higher delay levels correspond to larger PO networks and consequently to bigger differences between a priori SPs and SimSP. However trial 4 shows that this is not always the case: Since only few links are PO between New York and Salt Lake City (FIGURE 4(d)), a priori SPs cannot be very different from SimSP and in fact high SIs are recorded. Charts in (b) allow ranking the four heuristics according to the percentage of cases in which each of them provides the cheapest SP. Sometimes two heuristics can suggest the same path but the wider the PO network, the more 
the four a priori SPs differ from each other and from SimSP: In trial 1 (small PO network) in all 1,000 experiments (link state draws) the a priori SPs coincide and are often equal to SimSP, whereas in trials 59 very few overlaps occur. No heuristic method definitely outperforms the others. Still the local search provides good results in all trials. In (c) the optimality (or not) of LocSP w.r.t. to other a priori SPs and SimSP is represented by the percentage cost differences with the other SPs. In (d) the same indicator is reported for GloSP. The comparison with SimSP shows that the absolute performance of LocSP and GloSP is good when delays of the same magnitude of undelayed travel times are considered (trials 6 and 7). In this case the cost of LocSP and GloSP does not exceed that of SimSP by more than 6-7\% on average. For higher delay levels the difference can be an order of magnitude larger. The good relative performance of the local search is confirmed: LocSP outperforms or at most has the same cost as all other a priori SPs. The difference between LocSP and the other a priori SPs tends to increase with the OD distance and the level of potential delay.

Cyclic paths emerge in trial 7 , which disappear when penalties of $25 \%$ of ordinary travel times are applied to backward links.

\section{CONCLUSIONS}

An approach has been presented suitable for route choice in transport networks subject to potential delays with unknown probability. Free flow and maximum worst case link travel times only are considered to be known. Decision making is based on the assumption that such knowledge is used to avoid links which cannot be optimal in any link state on the network. Path selection combines the three most common decision principles reported in literature - optimism, pessimism and regret - and disappointment. The paper focuses on a mathematical formulation of expected frustration which can represent different attitudes towards frustration. Two interpretations of the expected frustration are discussed leading to two heuristic methods referred to as global and local search. During the local search cyclic paths can arise; the problem is solved by applying penalties to geographically backward-oriented links.

The two methods are evaluated by Monte Carlo simulation for the US highway network. Different OD pairs and different levels of potential delay are considered. For common levels of delay the difference between the costs of the SPs identified by the two methods and that of the optimal solution is satisfactory given the high level of uncertainty involved in the route choice. The local search appears to outperform the alternative heuristic methods, particularly when the number of PO links connecting the origin and destination increases. The approach seems reasonable from a behavioural point of view (a crucial characteristic for the effectiveness of navigation assistance) and is promising as to the performance.

Further studies are needed to confirm such preliminary results. Computer-based experiments similar to those in (19) can test the attitude of travellers towards frustration in link-based route choice. Monte Carlo simulations in networks with real travel time data are needed analysing the influence of $\alpha_{1}, \alpha_{2}$ and $\beta$, and extending the comparison to other heuristic approaches (e.g. robust optimization or random path selection in PO networks). Furthermore, alternative methods to deal with the problem of loops in local search are to be investigated aiming to minimize the search time. Finally, the possibility of using the proposed expected frustration in assignment problems might be explored.

\section{REFERENCES}

1. Park, K., Bell, M.G.H., Kaparias, I. and Bogenberger, K. Learning user preferences of route choice behaviour for adaptive route guidance. IET Intelligent Transport Systems, Vol. 1, No. 2, 2007, pp. 159166.

2. Nicholson, A., Schmöcker, J.D., Bell, M.G.H. and Iida, Y. "Assessing Transport Reliability: Malevolence and User Knowledge". In The Network Reliability of Transport, M.G.H. Bell and Y. Iida (eds), 1-22, Pergamon, 2003.

3. Kaparias, I. Reliable Dynamic In-Vehicle Navigation. Ph.D. thesis, Centre for Transport Studies, Imperial College London, London, 2007. 
4. Hart, P.E., Nilsson, N.J. and Raphael, B. A formal basis for the heuristic determination of minimum cost paths. IEEE Transactions on Systems Science and Cybernetics SSC4, Vol. 4, 1968, pp. 100-107.

5. Pallottino, S. and Scutellà, M.G. "Shortest Path Algorithms in Transportation Models: Classical and Innovative Aspects", University of Pisa, 1997.

6. Ding, B., Yu, J.X. and Qin, L. "Finding time-dependent shortest paths over large graphs". Presented at Proceedings of the 11th International Conference on Extending Database Technology: Advances in Database Technology, Nantes, France, 2008.

7. Yu, G. and Yang, J. On the robust shortest path problem. Computers \& operations research, Vol. 25, No. 6, 1998, pp. 457-468.

8. Hall, R.W. The Fastest Path through a Network with Random Time-Dependent Travel Times. Transportation Science, Vol. 20, No. 3, 1986, pp. 182-188.

9. Miller-Hooks, E. and Mahmassani, H. Path comparisons for a priori and time-adaptive decisions in stochastic, time-varying networks. European Journal of Operational Research, Vol. 146, No. 1, 2003, pp. 67-82.

10. Miller-Hooks, E.D. and Mahmassani, H.S. Least possible time paths in stochastic, time-varying networks. Computers \& operations research, Vol. 25, No. 12, 1998, pp. 1107-1125.

11. Nielsen, L.R., Pretolani, D. and Andersen, K.A. Bicriterion Shortest Paths in Stochastic TimeDependent Networks. Multiobjective Programming and Goal Programming: Theoretical Results and Practical Applications, Vol. 618, 2009, pp. 57-67.

12. Golledge, R.G. and Gärling, T. "Spatial Behavior in Transportation Modelling and Planning". In Transportation Engineering Handbook, K. Goulias (ed), 2001.

13. Golledge, R.G. and Stimson, R.J. Spatial behavior: a geographic perspective. Guilford Press, New York, 1997.

14. Schmöcker, J.D. "On Decision Principles for Routing Strategies Under Various Types of Risks". In Security and Environmental Sustainability of Multimodal Transport, M.G.H. Bell, S.H. Hosseinloo and U. Kanturska (eds), Springer, Dordrecht, The Netherlands, 2010.

15. Savage, L.J. The foundations of statistics. Wiley, New York,, 1954.

16. Bell, D.E. Disappointment in Decision Making under Uncertainty. Operations Research, Vol. 33, No. 1, 1985, pp. 1-27.

17. Loomes, G. and Sugden, R. Disappointment and Dynamic Consistency in Choice under Uncertainty. The Review of Economic Studies, Vol. 53, No. 2, 1986, pp. 271-282.

18. Coricelli, G. and Rustichini, A. Counterfactual thinking and emotions: regret and envy learning. Philosophical Transactions of the Royal Society B-Biological Sciences, Vol. 365, No. 1538, 2010, pp. 241-247.

19. Chorus, C.G., Arentze, T.A. and Timmermans, H.J.P. A random regret-minimization model of travel choice. Transportation research. Part B, Methodological, Vol. 42, No. 1, 2008, pp. 1-18.

20. Chorus, C.G. A New Model of Random Regret Minimization. European Journal of Transport and Infrastructure Research, Vol. 10, No. 2, 2010, pp. 181-196.

21. Chorus, C.G. Regret theory-based route choices and traffic equilibria. Transportmetrica, Forthcoming, pp. 1-15.

22. Kaparias, I., Bell, M.G.H., Bogenberger, K. and Chen, Y. An approach to time-dependence and reliability in dynamic route guidance. Transportation Research Record, Vol. 2039, 2007, pp. 32-41.

23. Bell, M.G.H. Hyperstar: A multi-path Astar algorithm for risk averse vehicle navigation. Transportation research. Part B, Methodological, Vol. 43, No. 1, 2009, pp. 97-107.

24. Fonzone, A., Schmöcker, J.D. and Bell, M.G.H. "Potentially optimal paths and route choice in networks with arc delays". Presented at 5th IMA Conference on Mathematics in Transport, London, UK, 12-14 April, 2010.

25. Chorus, C.G., Arentze, T.A. and Timmermans, H.J.P. Spatial choice: a matter of utility or regret? Environment and planning. B, Planning \& design, Vol. 36, No. 3, 2009, pp. 538-551.

26. Loomes, G. and Sugden, R. Regret theory: An alternative theory of rational choice under uncertainty. Economic Journal, Vol. 92, 1982, pp. 805-824. 
27. Fox, C.R. and See, K.E. "Belief and Preference in Decision Under Uncertainty". In Thinking: Psychological Perspectives on Reasoning, Judgement and Decision Making, D. Hardman and L. Macchi (eds), John Wiley \& Sons, Ltd., 2003.

28. Simon, H.A. Invariants of human behavior. Annual Review of Psychology, Vol. 41, No. 1, 1990, pp. $1-19$. 


\section{LIST OF TABLES}

TABLE 1 Specifications for different types of route choice

\section{LIST OF FIGURES}

FIGURE 1 Influence of parameters $\alpha_{1}, \alpha_{2}, \beta$ on route choice - (a) anticipated cost of link $a$, (b) expected disappointment of link $a$, (c) expected regret of links $a$ and $b$, (d-e) expected frustration of link $a$, (f) link chosen by local search

FIGURE 2 Influence of network description on route choice 18

FIGURE 3 Outline of Monte Carlo simulation 19

FIGURE 4 US highways case study - (a) Origins and Destinations, (b) PO networks between New York and Los Angeles for different levels of delay (trial 6: black links with dots, trial 7: black links with and without dots, trial 8: all black links and bold gray ones, trial 9: all black and gray links), (c) LocSP and GloSP (in black, dots identify links in GloSP but not in LocSP) in trial 7, (d) PO network in trial 4

FIGURE 5 Performance of the local and global search for different OD pairs - (a) Similarity Index, (b) \% of cases in which the method provides the best a priori path, (c) \% relative cost difference between LocSP and other shortest paths, (d) \% relative cost difference between GloSP and other shortest paths

FIGURE 6 Performance of the local and global search for different levels of potential delay - (a) Similarity Index, (b) \% of cases in which the method provides the best a priori path, (c) \% relative cost difference between LocSP and other shortest paths, (d) \% relative cost difference between GloSP and other shortest paths 
TABLE 1 Specifications for different types of route choice

\begin{tabular}{|c|c|c|}
\hline & Short-sighted & Far-sighted \\
\hline Not considering regret & \multicolumn{2}{|c|}{$\hat{A}_{a^{+}}^{+}=\{\}$} \\
\hline \multirow{2}{*}{ Isolated } & $\lambda=0$ & $\lambda=1$ \\
& $\hat{A}_{a^{+}}^{+}=\{a\}$ & $\hat{A}_{a^{+}}^{+}=\{a\}$ \\
\hline \multirow{2}{*}{ Comparative } & $\lambda=0$ & $\hat{A}_{a^{-}}=A_{a^{-}}^{+}$ \\
$\hat{A}_{a^{-}}^{+}=A_{a-}^{+}$ \\
\hline
\end{tabular}




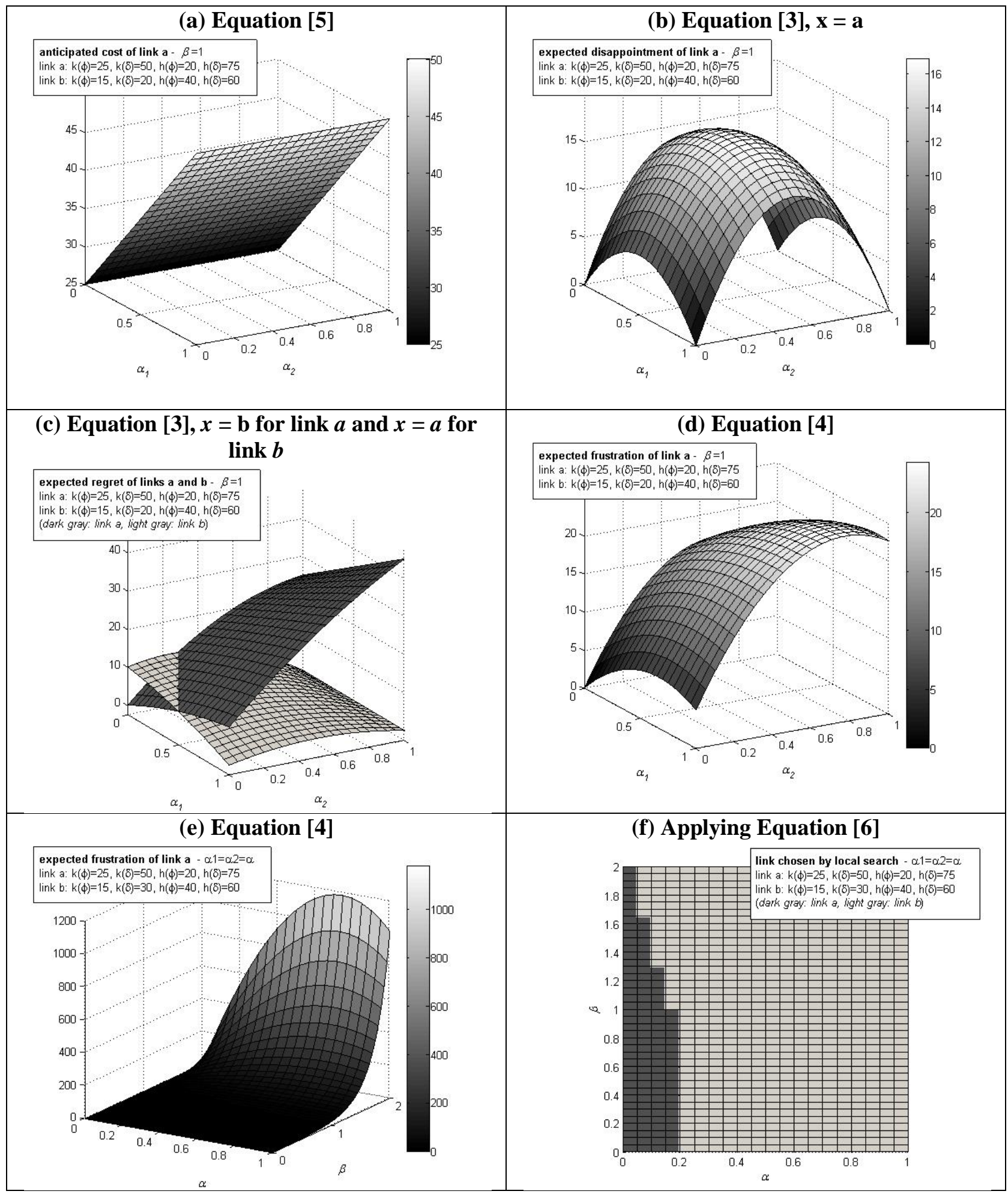

FIGURE 1 Influence of parameters $\alpha_{1}, \alpha_{2}, \beta$ on route choice - (a) anticipated cost of link $a$, (b) expected disappointment of link $a$, (c) expected regret of links $a$ and $b$, (d-e) expected frustration of link $a$, (f) link chosen by local search 


\begin{tabular}{|c|c|c|c|c|c|c|c|c|c|c|}
\hline \multicolumn{2}{|c|}{ Network } & \multicolumn{8}{|c|}{ Network 2} & \\
\hline & Link & $k_{x}(\phi)$ & $k_{x}(\delta)$ & $h_{x^{+}}(\phi)$ & $h_{x^{+}}(\delta)$ & $E\left(k_{x}\right)$ & $E\left(r_{x}\right)$ & $k_{x}^{\prime}$ & $E\left(g_{p_{x}}\right)$ & $g_{p_{x}}^{\prime}$ \\
\hline \multirow{2}{*}{$\begin{array}{c}\text { Network } \\
1\end{array}$} & $\mathrm{a}$ & 20 & 55 & 35 & 70 & 48.00 & 2.68 & 50.68 & 93.50 & 96.18 \\
\hline & b & 35 & 45 & 50 & 55 & 43.00 & 2.14 & 45.14 & 94.50 & 96.64 \\
\hline \multirow{4}{*}{$\begin{array}{c}\text { Network } \\
2\end{array}$} & $\mathrm{a}$ & 20 & 55 & 35 & 70 & 48.00 & 2.74 & 50.74 & 93.50 & 96.24 \\
\hline & $\mathrm{b}^{\prime}$ & 25 & 30 & 60 & 70 & 29.00 & 1.71 & 30.71 & 92.00 & 93.71 \\
\hline & b" & 10 & 15 & 50 & 55 & 14.00 & 0.82 & 14.82 & 65.50 & 66.32 \\
\hline & $b^{\prime}+b^{\prime \prime}$ & 35 & 45 & 50 & 55 & 43.00 & 2.53 & 45.53 & & \\
\hline
\end{tabular}

FIGURE 2 Influence of network description on route choice 
Define the OD pair, the undelayed and delayed travel times, and the number $n$ of the experiments for the trial

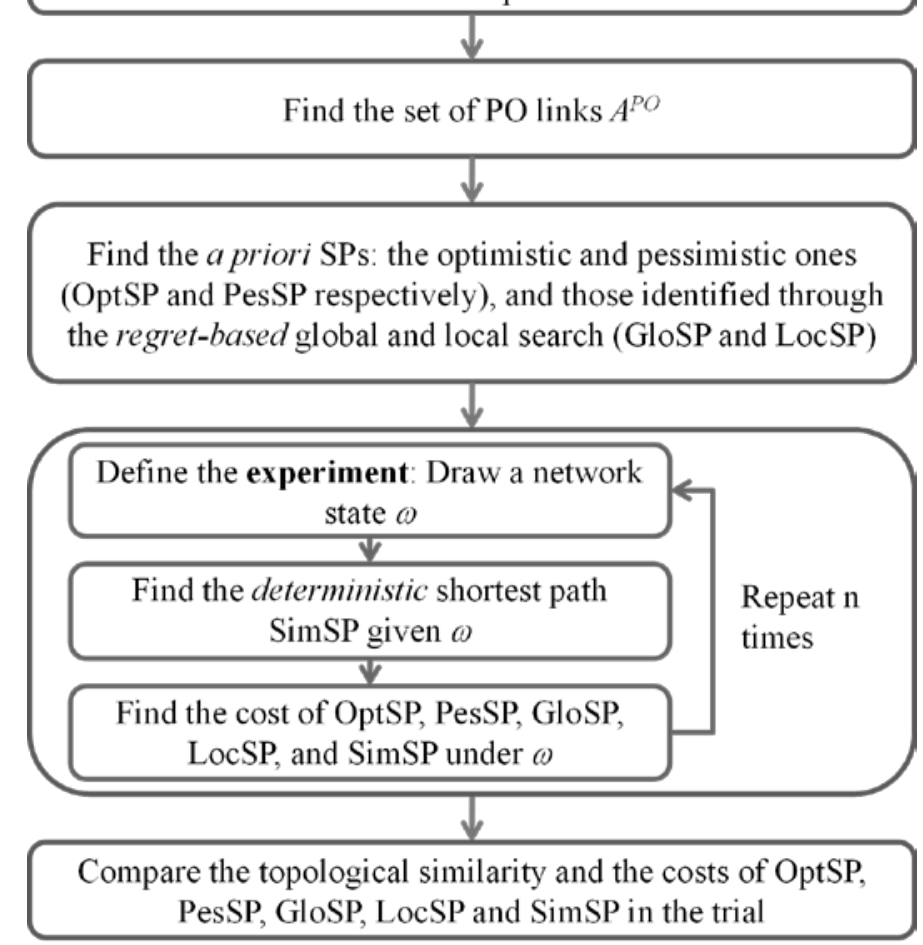

FIGURE 3 Outline of Monte Carlo simulation 


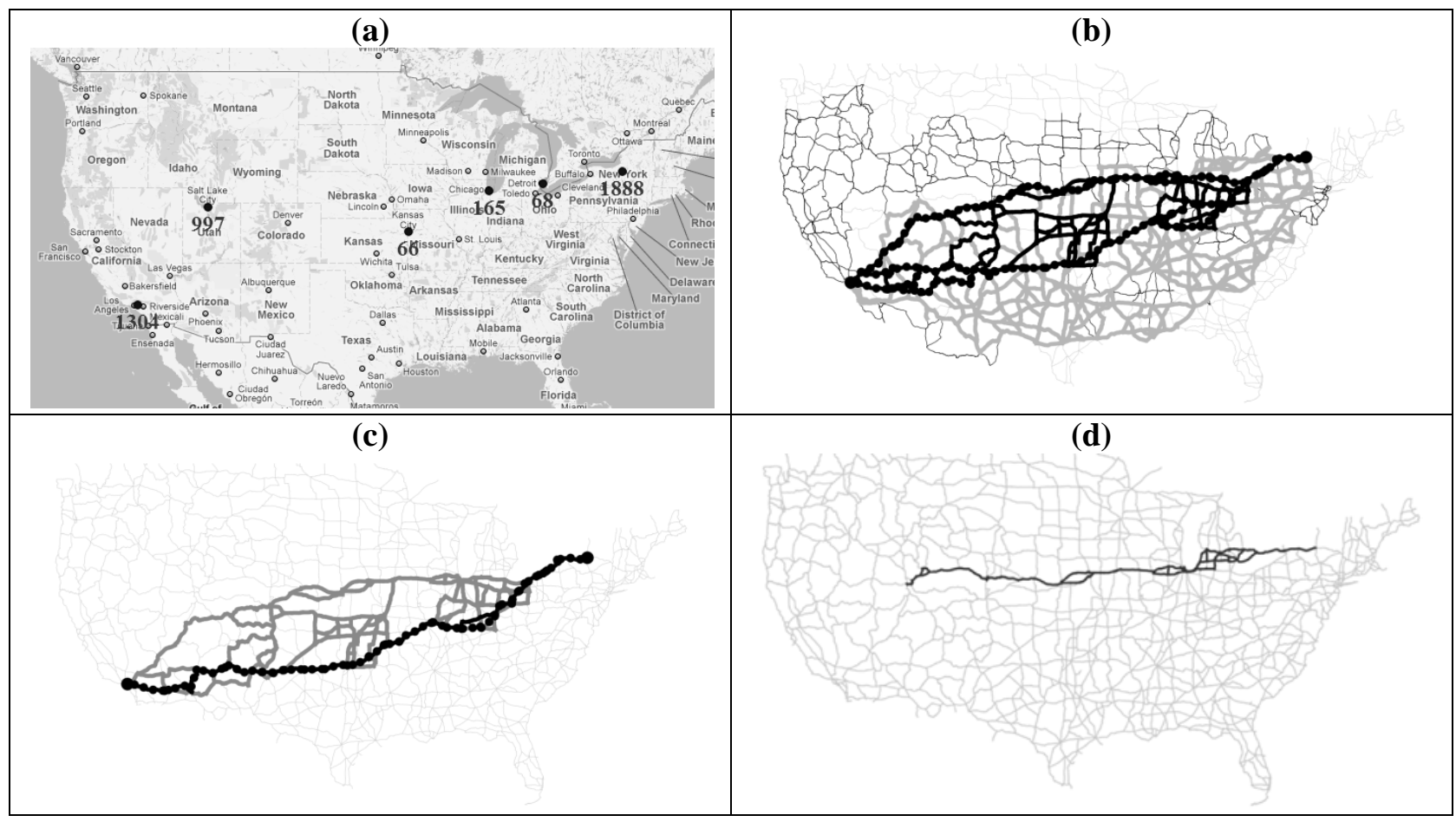

FIGURE 4 US highways case study - (a) Origins and Destinations, (b) PO networks between New York and Los Angeles for different levels of delay (trial 6: black links with dots, trial 7: black links with and without dots, trial 8: all black links and bold gray ones, trial 9: all black and gray links), (c) LocSP and GloSP (in black, dots identify links in GloSP but not in LocSP) in trial 7, (d) PO network in trial 4 


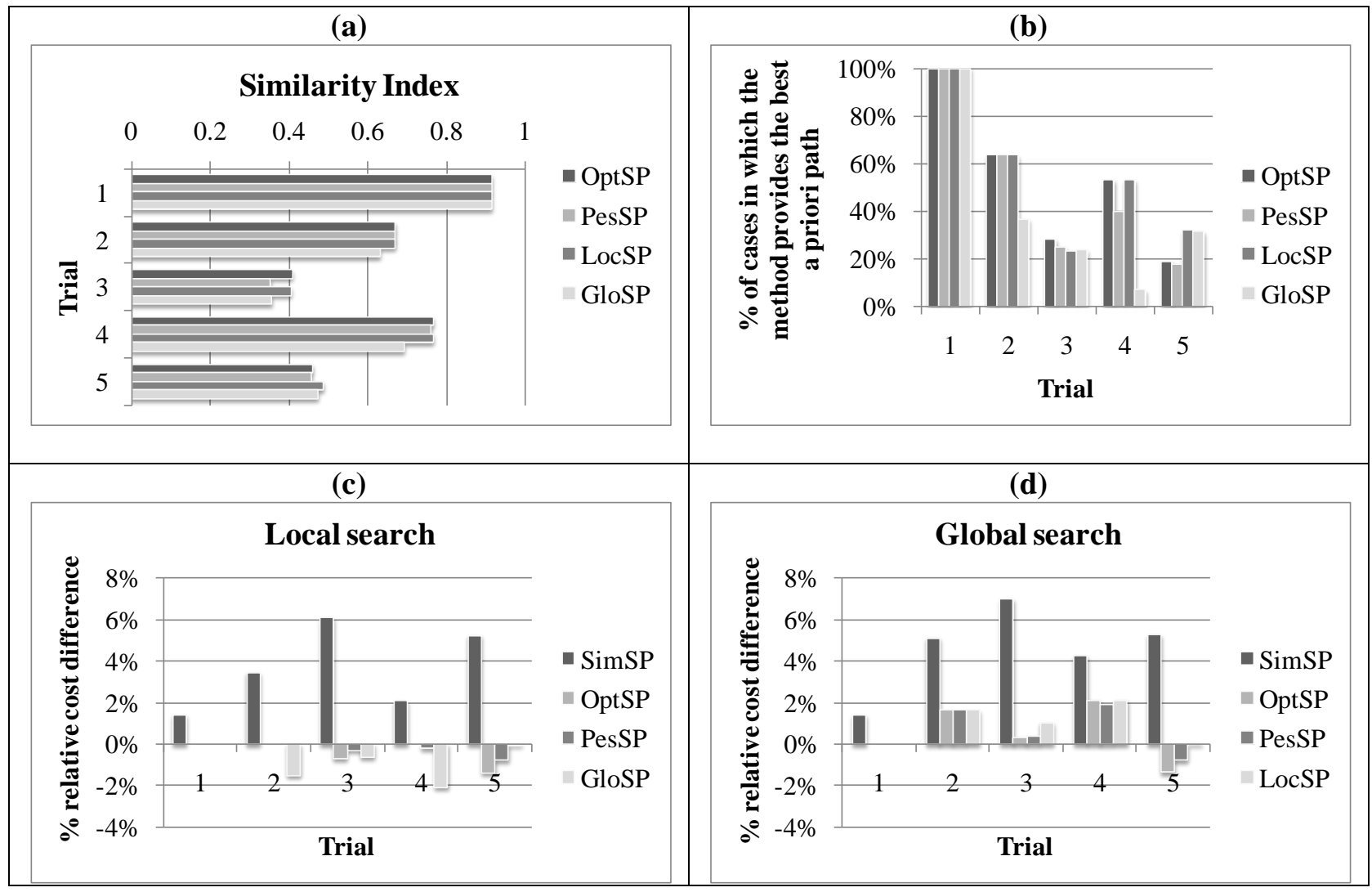

FIGURE 5 Performance of the local and global search for different OD pairs - (a) Similarity Index, (b) \% of cases in which the method provides the best a priori path, (c) \% relative cost difference between LocSP and other shortest paths, (d) \% relative cost difference between GloSP and other shortest paths 


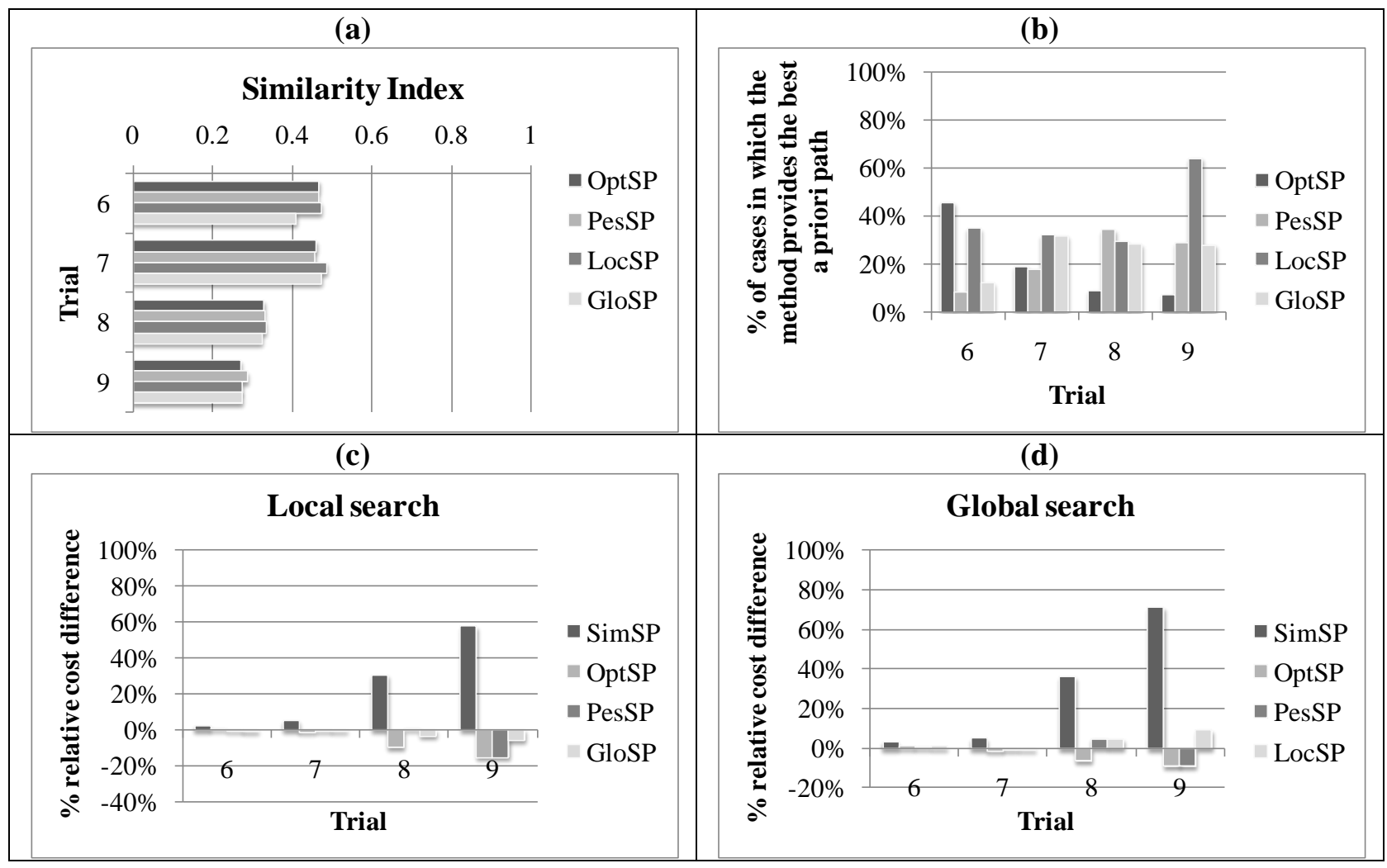

FIGURE 6 Performance of the local and global search for different levels of potential delay - (a) Similarity Index, (b) \% of cases in which the method provides the best a priori path, (c) \% relative cost difference between LocSP and other shortest paths, (d) \% relative cost difference between GloSP and other shortest paths 